\title{
Aplicación del software GeoGebra en prácticas matemáticas bajo una metodología constructivista
}

\section{GeoGebra application of software in mathematics practices under a constructivist methodology}

\author{
Sarmiento-Espinoza, William*; Luna-Altamirano, Kleber \\ Universidad Católica de Cuenca \\ Cuenca, C.P. 010150, Ecuador \\ *wsarmiento@ucacue.edu.ec
}

\begin{abstract}
Resumen
Las prácticas realizadas tienen un doble objetivo, primero: que el estudiante a través del conocimiento aporte con su creatividad un instrumento medible, que facilite el entendimiento en un menor tiempo; el segundo: generar a través de la práctica ejercicios y problemas a partir de modelos estudiados que logren destrezas significativas capaces de aplicar al campo administrativo. Este trabajo tiene aplicación en temas como funciones, derivadas e integrales, las mismas que son mejoradas año tras año y evidencia la trascendencia de tal aplicación. Los datos recogidos en las pruebas exploratorias y las encuestas se analizaron utilizando estadística descriptiva. Los hallazgos de estudio fueron: los conocimientos, la creatividad y el trabajo en grupo considerados como las capacidades más apreciadas. Por lo tanto, el estudio aportó evidencias favorables para aplicar el software GeoGebra, como herramienta didáctica en las prácticas bajo una metodología constructivista.
\end{abstract}

Palabras clave: Matemática, metodología, prácticas, software.

\begin{abstract}
The practices have a dual purpose, the student through knowledge contribution with their creativity a measurable instrument to facilitate understanding in a shorter time, the second generated through practice exercises and problems from studied models that achieve significant skills able to apply to the administrative field; This work has application in topics as functions, derivatives and integrals thereof which are improved year after year which demonstrates the importance of such an application. The data collected in exploratory tests and surveys were analyzed using descriptive statistics. The findings of the study were: knowledge, creativity and teamwork considered the most prized capabilities. Therefore, the study provided evidence favorable to apply the GeoGebra software, as a teaching tool in a constructivist methodology practices.
\end{abstract}

Key words: Mathematics, methodology, practice software.

\section{Introducción}

Para nadie es desconocido que uno de los problemas principales de deserción estudiantil, incluido con otros aspectos relacionados al entorno del estudiante, son las asignaturas donde más del $50 \%$ resultan ser prácticas, el adolescente desde temprana edad, mantiene cierta resistencia a tener un hábito de estudio que posibilite generar destrezas, que poco a poco conlleven a obtener prerrequisitos necesarios para aplicarlos en conocimientos nuevos.

Una de estas asignaturas es la Matemática, donde algunos procedimientos resultan tediosos y en otras incomprensibles, para ello se ha visto que la aplicación de un software, cambia el entorno del estudiante a un ambiente nuevo de participación, donde se descubren hechos con mucha facilidad y rapidez, provocando que este conocimiento sea significativo y que a la vez sea el punto de partida para generar aplicaciones directas al campo de estudio, donde se la involucra. El problema de investigación planteado es: Implementar prácticas con GeoGebra dirigidas para los estudiantes del segundo y tercer ciclo de la Unidad Académica de Administración de la Universidad Católica de Cuenca. Estas tienen una metodología que coadyuvan al estudiante, a más, de estructurar por si mismos la práctica, disponer de miles de ejercicios y problemas, que hacen que se pueda consolidar destrezas que luego serán aplicadas al campo administrativo.

La metodología planteada en cada una de ellas genera responsabilidades serias en el estudiante de tal manera que se sienta comprometido a consolidar su propio conocimiento, dejando de lado la deshonestidad académica 
y realizando un trabajo real en clase, donde predomine la creatividad y el trabajo grupal. Las dificultades en el aprendizaje de las matemáticas, es una problemática a nivel nacional. En el Ecuador luego de la aplicación de pruebas, se obtuvieron promedios muy bajos. Aunque estas pruebas no siempre se ajustan a la realidad de cada región del país, podemos considerarlas como una referencia de lo que está sucediendo en el proceso de aprendizaje de las matemáticas y dentro de estas del Cálculo diferencial e integral.

En la actualidad, el Internet ha revolucionado la concepción tradicionalista, haciendo que el estudiante se involucre en el mundo virtual. Entendemos que es ahora el momento de cambiar, de romper paradigmas, utilizar otras metodologías con herramientas apropiadas para que el estudiante palpe la realidad de los conocimientos y los haga suyos.

Esta propuesta de implementar y aplicar prácticas en funciones, derivadas e integrales, nace de las aulas con los mismos estudiantes, como actores importantes en la construcción del conocimiento. De acuerdo a datos estadísticos se puede afirmar que los estudiantes de los primeros ciclos de la Unidad Académica de Administración, no llegan con un manejo adecuado de los elementos básicos del álgebra elemental. Además con mucha dificultad pueden graficar una función o expresión algebraica, se puede considerar que sus destrezas de deducción e inducción son limitadas. El razonamiento lógico es mínimo.

Cuando se revisa la descomposición factorial, en las pruebas de diagnóstico se nota que las destrezas no fueron nunca alcanzadas en su nivel medio, peor aún óptimo, las funciones ni siquiera son vistas por algunos estudiantes, no conocen otra herramienta más que el papel y lápiz y ningún otro recurso que motive su interés por aprender. Lo mayoría de los Catedráticos del área Cuantitativa, utilizan metodología tradicional para sus clases. El resultado con esta metodología, es una marcada monotonía en las clases impartidas. La creatividad de los estudiantes, como resultado de esta forma de enseñar es nula, la riqueza del trabajo desaparece por completo en el aula, dándose una marcada intención de deshonestidad académica. En este contexto se pregunta:

¿Cuáles son las mejoras que se consiguen en el aprendizaje significativo de los estudiantes al trabajar en un ambiente de prácticas experimentales?

¿Qué aspectos se deben considerar para proponer clases prácticas de matemáticas basadas en el uso de software?

¿Cuál es el software educativo que nos permitirá mejorar el aprendizaje de las funciones, derivadas e integrales?

¿De qué manera el uso del software conseguirá facilitar la aplicación al campo administrativo?

\section{Estado del arte}

La tecnología educativa puede generar puentes a partir de diferentes posiciones teóricas entre ellas el constructivismo. (Trigo, 2003) Es por ello que el profesor frente a una herramienta tecnológica deberá identificar rigurosamente las características de la misma para la implementación del paradigma educativo deseado. El principio básico de la teoría constructivista (Díaz Barriga Arceo y Rojas, 2002) es que el aprendizaje humano se construye. La mente humana elabora nuevos conocimientos a partir de anteriores. Un supuesto también básico es que las personas aprenden cuando pueden controlar su aprendizaje y que tienen conciencia de este control.

De acuerdo a las investigaciones de los últimos años, se considera al computador como un apropiado medio creativo, que apoya al aprendizaje constructivista. Una característica del profesor constructivista es motivar a sus alumnos a usar el ordenador para actividades escolares. Existen diferentes aplicaciones informáticas en este sentido entre las cuales se caracterizan las wikis, los blogs y las redes sociales (Díaz Barriga Arceo y Rojas, 2002). Permiten al alumno exponerse a un entorno mucho más amplio y acceder de forma ilimitada a conocimientos. Estas herramientas lo que provocan es que el alumno pase del papel de consumistas al papel de productores de información.

Los puentes tecnológicos, permiten el control sobre el aprendizaje para la construcción de nuevo conocimiento. Brinda al ser humano la capacidad de crear, compartir y dominar dicho conocimiento (Díaz Barriga Arceo y Rojas, 2002). Varios autores destacan las características de las TIC que son soporte para el aprendizaje. Otra característica que se vincula con la tecnología es el aprendizaje significativo. Según (Ausubel, 1983), p.32 “con el aprendizaje significativo, los nuevos conocimientos se incorporan en forma sustantiva en la estructura cognitiva del alumno". (Ausubel, 1983) indica "Si tuviese que reducir toda la psicología educativa a un solo principio, enunciaría este: El factor más importante que influye en el aprendizaje es lo que el alumno ya sabe. Averígüese esto y enséñese consecuentemente". Otro de los factores que influye directamente en el aprendizaje es la creatividad. Gámez [1998], admite a la creatividad como la capacidad de crear, producir, formar, dar nacimiento, dar origen o representar por primera vez, y es que la creatividad es uno de los más grandes y nobles principios indispensables en todo proceso o enseñanzaaprendizaje, para contribuir al desarrollo del ser humano como una unidad Bio-Psico-Social-Trascendente.

En lo referente al uso tecnológico, (Balderas, 2013) indica "la evolución que ha experimentado el software nos ofrece nuevas formas de enseñar, aprender y hacer matemática, brindando amplias posibilidades didácticas". De igual manera destacan "la capacidad de esta herramienta para lograr la interacción del alumnado con situaciones de aprendizaje que lo conduzcan a construir conocimientos que le permitan tener una visión más amplia del contenido matemático" (Maryianela, 2005). De allí, el interés de investigar sobre la aplicación de estrategias donde se usa el software como herramienta para lograr destrezas con criterio de desempeño que permitan mejorar sustancialmente el proceso de interaprendizaje del cálculo diferencial e integral. "Con los avances tecnológicos se han desarrollado 
muchos programas en los cuáles se proporcionan medios para la enseñanza de la matemática; sin embargo, el docente debe saber aprovecharlos para generar situaciones que permitan al alumnado construir un conocimiento más significativo" (Ángel y Guillermo Bautista, 2013). La idea es que el estudiante utilice la tecnología computacional como una herramienta de apoyo al proceso de interaprendizaje, para lograr comprender y entender mejor los conceptos matemáticos y reflejarlos en la solución de problemas, "no pretende la instrucción del alumno sino, viabilizar la construcción del conocimiento, para que los estudiantes aprendan con ellas no de ellas" Williner [2011]. En tal sentido el software se usó con fines didácticos para facilitar estrategias de interaprendizaje relacionadas con el tema prácticas experimentales para funciones, derivadas e integrales.

\section{Metodología}

"La enseñanza eficaz de las matemáticas requiere comprender lo que los estudiantes conocen y necesitan aprender $\mathrm{y}$, en consecuencia, les desafía y apoya para aprender bien los nuevos conocimientos" (NCTM, 2000, Principio de la Enseñanza). En este contexto se origina al principio de cada una de las prácticas un estudio dirigido que metódicamente la llamaremos clase dirigida. Consiste en indicar a los estudiantes la forma como realizamos la práctica a través del software GeoGebra, y una vez que se ha consolidado esta información entonces el estudiante comienza a deducir los resultados para que los mismos sean el inicio de nuevos conocimientos.

Esta apropiación del conocimiento hace que los estudiantes se identifiquen con los problemas propuestos, originen discusiones que conllevan a la solución de problemas; sin embargo en la marcha se van detectando inconvenientes en el grupo, y es el momento en que el docente aplica el çontrato didáctico". El cual sirve para describir y explicar las obligaciones o normas no explícitas que rigen las interacciones entre el profesor y los alumnos en el aula. De igual forma ayuda a que su comportamiento sea estrictamente de servicio al grupo, para mantener siempre el respeto ante hallazgos que se van detectando en el transcurso de la actividad propuesta, Todos los criterios emitidos por el grupo son importantes en tanto y cuanto hagan crecer la comprensión, el análisis, el control de los resultados y la búsqueda para solucionar los problemas (Godino, 2009).

La aplicación del estudio dirigido dentro del aula, es una herramienta didáctica muy conveniente si se la concibe desde un punto de partida en la conformación de grupos de trabajo, mas no en las decisiones que se vendrán más adelante.

Cuando esté especificada la práctica, ese momento será especial para los estudiantes, pues consolidarán sus conocimientos y destrezas a través de un análisis serio que se refleja a través de las prácticas.

A modo de ejemplo se demuestra la siguiente práctica:
Dadas las ecuaciones de oferta y demanda determine el Excedente de los Consumidores y Productores bajo equilibrio del mercado. Consideremos si las ecuaciones de oferta y demanda fuesen lineales como se indica en la Fig. 1), en primer lugar se crea 4 deslizadores $\mathrm{m}, \mathrm{b}, \mathrm{m} 1$, b1 que significan la pendiente de la recta y el punto de corte con el eje y respectivamente, a continuación en la entrada se escribe: $\mathrm{f}(\mathrm{x})=\mathrm{mx}+\mathrm{b} ; \mathrm{g}(\mathrm{x})=\mathrm{m} 1 \mathrm{x}+\mathrm{b} 1$ en vista gráfica se observará la gráfica de las ecuaciones de oferta y demanda así,

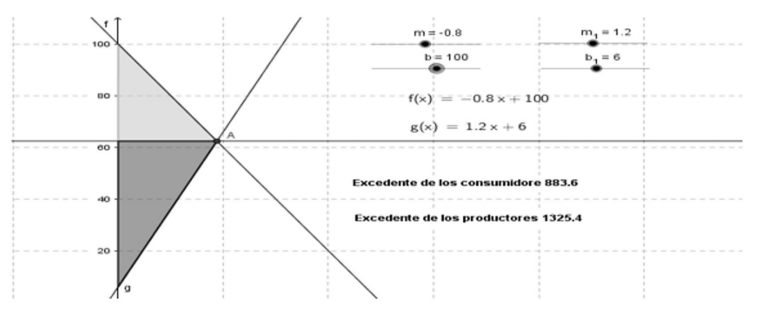

Figura 1. Excedente del consumidor y productor

Para determinar el excedente de los consumidores se escoge la opción intersección de dos objetos y se señala el punto de corte en las ecuaciones graficadas, GeoGebra llamará a ese punto $A$, luego se escribe en la entrada $h(x)=$ $y(A)$ apareciendo una función constante, por último en la entrada se escribe: IntegralEntre $[<$ Funcin $>,<$ Funcin $>,<$ ValorInicialdex $>,<$ Valor Finaldex $>$ ], en nuestro caso particular se sustituye función por $f(x)$, función por $h(x)$, valor inicial por 0 , Valor final por $x(A)$. Así: IntegralEntre $[f(x), h(x), 0, x(A)] \mathrm{y}$, GeoGebra sombreara y determinará el área correspondiente al excedente de los consumidores (Fig. 2).

Para el Excedente de los Productores se escribirá: IntegralEntre $[h(x), g(x), 0, x(A)]$ (Fig. 3) Note que los estudiantes podrán asegurarse de alcanzar esta destreza creando un sin número de ejemplos de este tipo. Se considera el caso de que la oferta y demanda sean funciones de segundo grado y lineales o una función de cualquier tipo, en verdad el procedimiento no difiere del anterior, únicamente cambiarán el número de deslizadores para que generen más problemas similares. Así, en este ejemplo en particular, el Área representa la ganancia total o lo que se denomina en Administración el Excedente de los Consumidores. En este ejemplo en particular, el área representa la ganancia total o lo que se denomina en Administración el Excedente de los Productores.

Una aplicación más se realizará utilizando funciones logarítmicas y funciones irracionales. La Ecuación de demanda para un producto es $p=80-\frac{70 q}{\sqrt{q^{2}}+3800}$, y la ecuación de oferta es $p=12 \ln (q+35) 40$

El Área comprendida entre $f(x)$ y $h(x)$, viene a representar el Excedente de los consumidores. De manera análoga el Área entre $h(x)$ y $g(x)$ representará el Excedente 


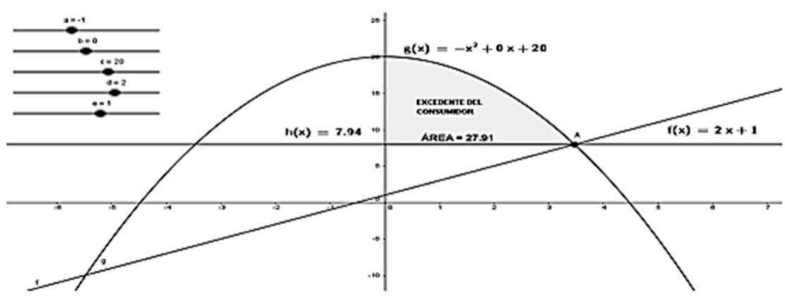

Figura 2. Excedente del Consumidor

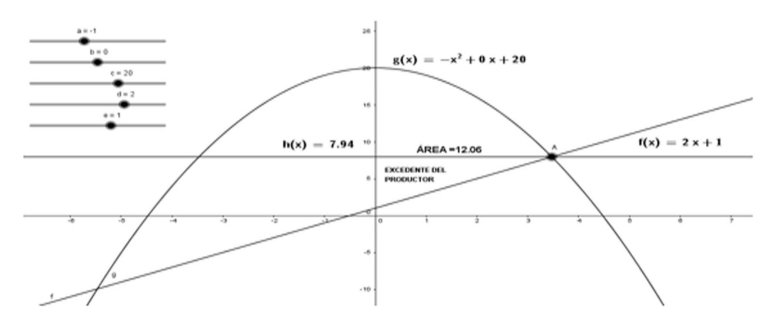

Figura 3. Excedente del Productor

de los productores. Como demuestra la fig 5:

\section{Resultados}

Los resultados muestran que el porcentaje de estudiantes que domina los aprendizajes requeridos (7-8) aumenta en el Ciclo Mar-Jul 2015, se observa también que aumenta de 0 al 3,2\% el porcentaje de quienes superan los aprendizajes requeridos; es importante señalar que en dicho año lectivo se elimina el porcentaje de estudiantes que están próximos a alcanzar los aprendizajes requeridos, lo manifestado se demuestra en la Fig. 6.

\section{Conclusiones y Recomenaciones}

Luego del análisis establecido en las diferentes prácticas, las mismas que apuntan a conseguir destrezas, el conocimiento no queda ahí, los estudiantes tratan de aplicar lo aprendido a situaciones reales aplicando al campo administrativo, por ejemplo, determinan niveles de producción donde la empresa empieza a generar utilidades, ubican precios que maximizan ingresos, cantidades que minimizan costos, puntos donde la empresa alcanza el equilibrio, Excedentes de los consumidores y productores.

La utilización del software matemático, permite realizar con mucha facilidad las deducciones de las fórmulas; la traslación y rotación de las funciones, determinación de extremos relativos, puntos de inflexión, áreas bajo una curva, y entre curvas; utilizando los deslizadores se pueden crear miles de miles ejercicios en el que el estudiante puede realizar conjeturas de alto nivel de razonamiento.

Una de las cualidades más importantes notadas a lo largo de este proceso de investigación fue la gran capacidad, habilidad y manejo del software. Su creatividad

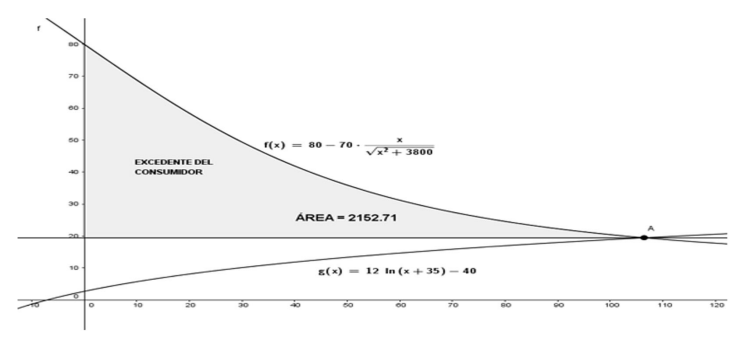

Figura 4. Excedente del Consumidor

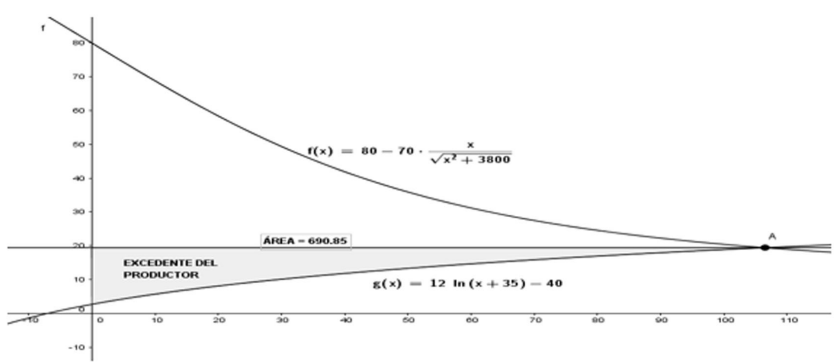

Figura 5. Excedente del productor

rompió barreras en los momentos de resolver problemas encaminados hacia el tema propuesto. Sin duda alguna los estudiantes se muestran muy entusiastas cuando se trata de compartir el conocimiento y al mismo tiempo aplicar sus descubrimientos.

Aunque no se comprobó un aumento notorio en promedios de notas, se tiene la satisfacción de haber propiciado un cambio en la metodología de la enseñanza matemática, de la experiencia se piensa que solo utilizando un software se podría cumplir con las expectativas de los estudiantes, pues estas prácticas aceleran de mejor manera la consecución de las destrezas propuestas.

La utilización de la tecnología, genera en los estudiantes seguridad, motivación, apoyo, mediación, afecto etc., lo que sin duda alguna fomenta la preparación individual y colectiva, el compañerismo, la creatividad y sobre todo afinidad con su profesor, lo cual propicia ambientes de trabajo de calidad, fomentando aprendizajes significativos.

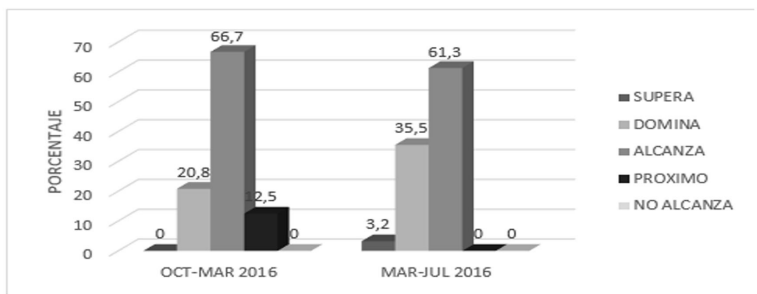

Figura 6. cuadro de notas 
El cambio de actividad también es fundamental en los estudiantes, el solo hecho de cambiar la metodología tradicional, hace que exista un clima armonioso, capaz de provocar conformidad para el trabajo; momentos fundamentales para establecer convenios y obligaciones con los estudiantes.

Los estudiantes de esta época revolucionaria de la tecnología, comienzan a satisfacer su curiosidad cognitiva a través de clases cibernéticas, lo que posibilita que se entre en este mundo globalizado por la competencia y se pruebe que también se puede crear. Esta creación permitirá interactuar con personas ajenas a la institución y demostrar las capacidades, generando ejercicios y problemas desarrollados a través del software GeoGebra.

Lo expuesto anteriormente, hace prevalecer la recomendación de seguir utilizando la tecnología y concretamente software matemático, para poder acercarse más a los estudiantes, tratando de establecer mediaciones importantes que a la larga serán ejes transversales cuyo objetivo final será tener estudiantes formados íntegramente y preparados para una eficaz inserción en nuestra sociedad.

\section{Referencias Bibliográficas}

Agudo, J. D. (2001). Curso de formación para equipos directivos. [Manual de software informático]. Madrid.

Ausubel, D. (1978). Psicología educativa, un punto de vista cognoscitivo. México: Editorial Trillas.

Ausubel, D. (1983). Teoría del aprendizaje significativo. (Fascículos del CEIF.)

Balderas, A. (2013). Didáctica de las matemáticas en internet.

Budnick, F. (1993). Matemáticas aplicadas para administración, economía y ciencias sociales. (Mc. Graw Hill, México.)

Dapía, A. C. (2008). Deconstrucción de la didáctica racionalista en el contexto de la formación docente. hacia una didáctica constructivista. Iberoamericana de Educación(45/3), 1-12.

Díaz Barriga Arceo, F., y Rojas, G. H. (2002). Estrategias docentes para un aprendizaje significativo. una interpretación constructivista. México: McGraw-Hill.

Delgado, J. (2001). Curso de formación para equipos directivos. (Ministerio de Educación y Ciencia, Madrid)

D. Zappalá, A. K., y Suchodolski, M. (2011). Las tic en el aula: estrategias didácticas.

Godino, J. D. (2009). Un enfoque ontosemiótico del conocimiento y la instrucción matemática. (Revista electrónica de educación matemática.)

Haeussler, E. (2003). Matemáticas para administración y economía. (Pearson, México décima edición.)

K. D Waits, F. (2007). Precálculo. (Pearson, México.)

Lipschutz, S. (1992). Matemática para computación. (McGraw Hill, México.)

Maryianela, M. G. (2005). El aprendizaje de funciones reales con el uso de un software educativo: una experiencia didáctica con estudiantes de educación de la ula-táchira. Acción Pedagogíca(14), 38-49.

Moreira, M. A. (2000). Aprendizaje significativo: Teoría y práctica. (Visor, México.)

Ángel y Guillermo Bautista, J. (2013). Didáctica de las matemáticas en la enseñanza superior.

Smith, L. G. (1990). Cálculo diferencial. (Uteha, México)

Toledo, C. G. (2008). Didáctica para profesores. (CODEU, Quito)

Trigo, L. M. S. (2003). Procesos de transformación de artefactos tecnológicos en herramientas de resolución de problemas matemáticos. (Boletín de la asociación matemática venezolana)

Webster, A. L. (2005). Estadistica aplicada a los negocios y a la economía. (Mc. Graw Hill, México.)

Williner, B. (2011). Estudio de habilidades matemáticas cuando se realiza actividades usando software especifico. (Revista Iberoamericana de educación matemática.)

y Gerardo Hernández Rojas, F. D. B. A. (2002). Estrategias docentes para un aprendizaje significativo: una interpretación constructivista. MCGraw Hill.

Recibido: 14 de junio de 2017

Aceptado: 28 de julio de 2017

Sarmiento-Espinoza, William: Docente de la Unidad Académica de Administración de la Universidad Católica de Cuenca, Licenciado en Administración, Contador Publico,Ingeniero Comercial, Especialista en Docencia Universitaria, Master en Didáctica de la Matemática.

Luna-Altamirano, Kleber: Docente de la Unidad Académica de Administración de la Universidad Católica de Cuenca, Licenciado en Economía y Finanzas, Economista, Magister en Administración de Empresas, Mención Recursos Humanos y Marketing.

Correo electrónico: klunaa@ucacue.edu.ec 
